\title{
Conservative Ultrathin Veneer Restorations with Minimal Reduction: A 5-year Follow-up Report
}

\author{
Carlos A Jurado ${ }^{1}$, Nicholas G Fischer ${ }^{2}$, Firas Mourad ${ }^{3}$, Jose Villalobos-Tinoco ${ }^{4}$, Akimasa Tsujimoto ${ }^{5}$
}

\begin{abstract}
Aim and objective: This case report shows how a feldspathic veneer with diagnostic wax-ups, subsequent mock-up, and reduction guides can lead to good patient esthetics and reports a 5-year follow-up.

Background: Conservative tooth preparation is important for the long-term success of adhesive dentistry as it has been shown that bonding to enamel is more predictable in obtaining better long-term success than dentin. To preserve enamel for optimal bonding, diagnostic wax-ups and the subsequent mock-up are the first tools in a restorative dentist's arsenal to find and address differences between current and ideal tooth proportions and also help toward an overall conservative approach. Reduction guides are recommended in order to provide adequate tooth reduction and prevent over-reduction.
\end{abstract}

Case description: This case report shows a 5 -year follow-up of feldspathic veneer restorations for a patient with excessive space among teeth, defective composite restorations on facial and incisal surfaces, and worn teeth. Veneers were delivered with conservative tooth preparation combining different tooth reduction guides.

Conclusion: This case report highlights the added benefits of tooth reduction guides and diagnostic wax-ups and the subsequent mock-up for long-term patient satisfaction.

Clinical significance: Conservative tooth preparation, reduction guides, and wax-ups may increase the life span of veneer restorations and demonstrate good esthetics at 5 years.

Keywords: Dental porcelain, Tooth preparation, Veneer.

The Journal of Contemporary Dental Practice (2020): 10.5005/jp-journals-10024-2946

\section{BACKGROUND}

Conservative restorative dentistry technique relies on bonding and adhesive luting to achieve adhesion..$^{1-3}$ In general, enamel bonding is stronger and longer lasting than dentin bonding. ${ }^{4}$ It is also known that caries-free, intact enamel is an ideal substrate for etched porcelain laminate veneers. ${ }^{5}$ Ceramic veneers have shown to be a long-lasting treatment when compared to composite veneers, and provide improved long-term esthetics. ${ }^{6,7}$ Clinical challenges associated with porcelain veneers still exist and include luting composite resin polymerization shrinkage and chipping, for example. ${ }^{8-10}$ Despite this, ceramic veneer restorations are still popular but their success relies on preparation design, ${ }^{11}$ adhesive bonding techniques and materials, ${ }^{12,13}$ and patient oral care practices. ${ }^{14}$ When it comes to preparation design, tooth reduction is necessary for ceramics to yield an optimal patient smile, but overreduction can reduce bond durability and restoration longevity due to dentin exposure. ${ }^{15}$

Recent advances in dental materials have made ultrathin ceramic veneers (thickness of around $0.1-0.5 \mathrm{~mm}$ ) able to bond to enamel with minimal to no tooth preparation. ${ }^{16,17}$ Many ceramics (such as lithium disilicate, lithium disilicate reinforced with zirconia, etc.) and others are currently available to clinicians. ${ }^{18-21}$ These ceramic materials have a relatively large concentration of a glass-based matrix that produces excellent esthetics and durable adhesion to resin cements when treated with appropriate adhesive treatments. ${ }^{22}$ In addition, conservative tooth reduction provides better mechanical properties - and so longer lasting restorationwhen resin cements are bonded to underlying enamel. ${ }^{7,23-25} \mathrm{Good}$ long-term survival rates and low failure rates have been reported for enamel-bonded ceramic veneers. ${ }^{26,27}$
${ }^{1}$ Clinical Digital Dentistry, AT Still University Arizona School of Dentistry and Oral Health, Mesa, Arizona, USA

${ }^{2}$ Minnesota Dental Research Center for Biomaterials and Biomechanics, University of Minnesota School of Dentistry, Minneapolis, Minnesota, USA

${ }^{3}$ Department of Care Planning and Restorative Sciences, University of Mississippi Medical Center School of Dentistry, Jackson, Mississippi, USA

${ }^{4}$ Department of Oral Rehabilitation. Autonomous University of Queretaro School of Dentistry, Queretaro, Queretaro, Mexico

${ }^{5}$ Department of Operative Dentistry, Nihon University School of Dentistry, Tokyo, Japan

Corresponding Author: Nicholas G Fischer, Minnesota Dental Research Center for Biomaterials and Biomechanics, University of Minnesota School of Dentistry, Minneapolis, Minnesota, USA, Phone: +1612-625-0950, e-mail: Fisc0456@umn.edu

How to cite this article: Jurado CA, Fischer NG, Mourad F, et al. Conservative Ultrathin Veneer Restorations with Minimal Reduction: A 5-year Follow-up Report. J Contemp Dent Pract 2020;21(11): 1293-1297.

Source of support: Nil

Conflict of interest: None

Preparation of ceramic veneers can be a challenge for clinicians with little experience, which may cause the restoration to fail. To aid in this process, diagnostic wax-ups are fundamental ${ }^{28}$ because they show differences between the existing and ideal tooth measurements. ${ }^{29-31}$ The wax-up can also be used as a diagnostic mock-up for evaluation. In addition, the diagnostic wax-up

( ) Jaypee Brothers Medical Publishers. 2020 Open Access This article is distributed under the terms of the Creative Commons Attribution 4.0 International License (https://creativecommons.org/licenses/by-nc/4.0/), which permits unrestricted use, distribution, and non-commercial reproduction in any medium, provided you give appropriate credit to the original author(s) and the source, provide a link to the Creative Commons license, and indicate if changes were made. The Creative Commons Public Domain Dedication waiver (http://creativecommons.org/publicdomain/zero/1.0/) applies to the data made available in this article, unless otherwise stated. 


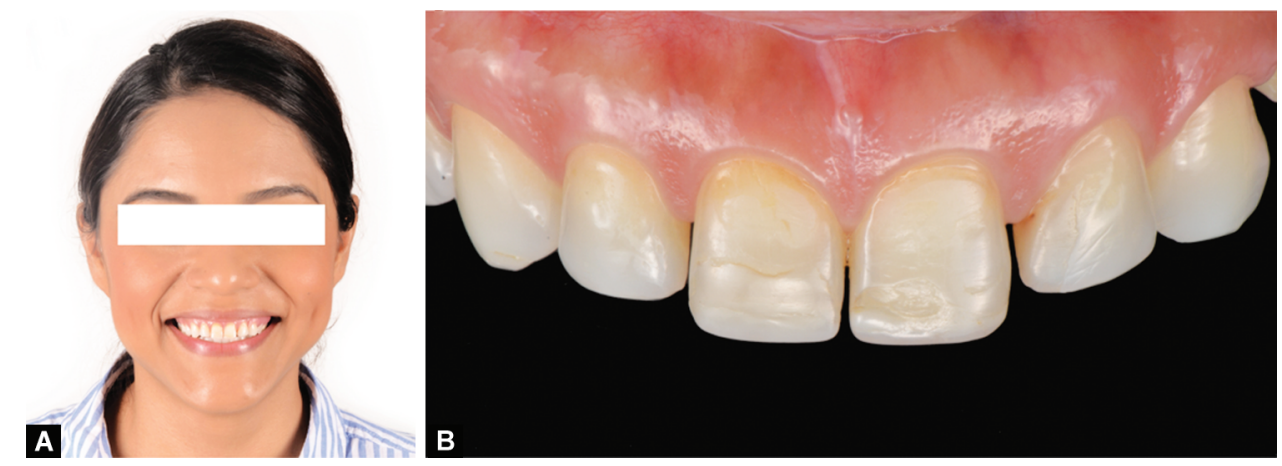

Figs $1 \mathrm{~A}$ and $\mathrm{B}$ : (A) Initial smile; (B) Initial intraoral

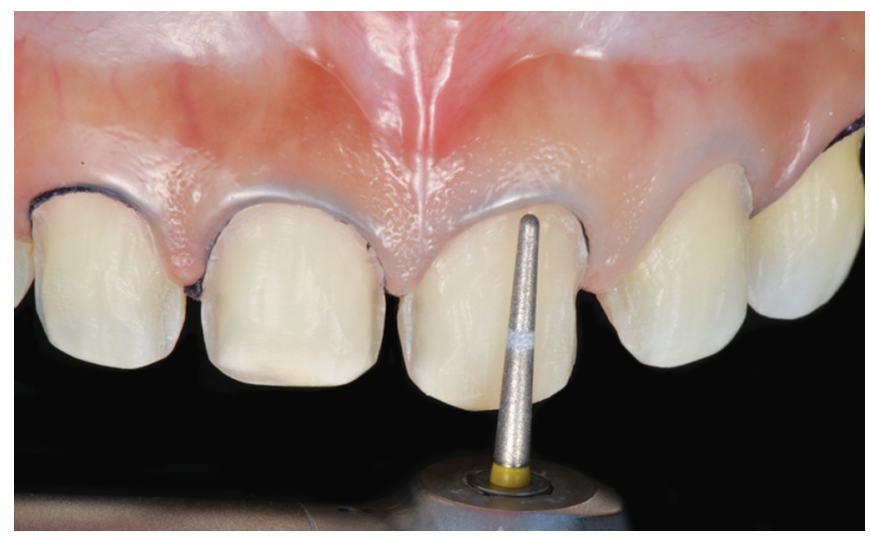

Fig. 2: Tooth preparations

functions can be used to make preparation reduction guides, again potentially useful for clinicians with less experience. ${ }^{32}$ Final tooth preparation can be made on the diagnostic mock-up in the oral cavity with a putty guide matrix. In short, mock-ups with provided initial groove depths help the clinician perform conservative veneer preparations because less enamel removal is needed. . $^{33,34}$

This clinical report describes a method for ceramic veneers using a diagnostic mock-up, followed by controlled tooth preparation using different reduction guides for an overall conservative approach. A 5-year follow-up is also reported with retained results.

\section{Case Description}

A 34-year-old patient (female) presented to the dentist with the chief complaint of "I do not like my smile" (Fig. 1). The patient presented at the Oral Rehabilitation Department at the Autonomous University of Queretaro School of Dentistry, Mexico. After initial assessment, the diagnosis was excessive space among teeth \#6 to \#7, \#7 to \#8, \#8 to \#9, \#10 to \#11, defective composite restorations on facial and incisal surfaces of \#8 and \#9, and worn teeth on $\# 6, \# 8, \# 9, \# 10$, and \#11. A treatment plan consisting of a combination of orthodontics, restorative treatment, and tooth bleaching was discussed. Endodontic treatment was not needed. The patient declined the bleaching treatment and the orthodontic treatment. A diagnostic wax-up (GEO Classic Renfert) followed by fabrication of a mock-up guide (Bisacril Telio CS C\&B Ivoclar Vivadent) was performed. The patient was satisfied. They agreed upon the course of action consisted of porcelain veneers on teeth $\# 6,7,8,9,10$, and 11 .

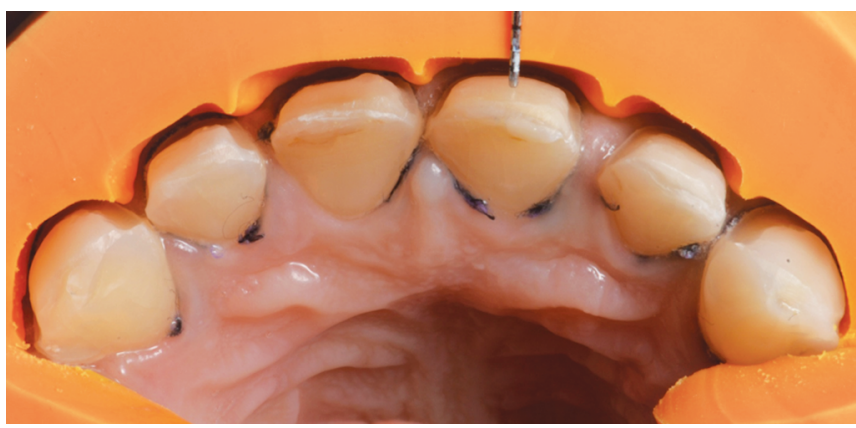

Fig. 3: Facial reduction guide

At the following clinical appointment, retraction cord \#000 (Ultrapak, Ultradent) was placed and conservative tooth preparation was performed using reduction guides to evaluate incisal, facial, and two plane reduction (Figs 2 and 3). The final preparations were polished with polishing discs (Soft-lex TX Disc, 3M) (Fig. 4A). For optimal results, cords were first packed at \#00 and then at \#0 (Ultrapak, Ultradent Products Inc.). Type IV stone (Fujirock, GC America Inc.) was used after the final impression (polyvinylsiloxane material; Elite HD, Zhermack) to make cast and dies. Feldspathic porcelain (IPS e-max, Ivoclar Vivadent) was used to create ceramic veneers.

Try-in allowed contours and margins to be evaluated, and the patient requested to proceed with the final bonding procedures. The teeth were cleaned with a pumice paste and chlorhexidine gluconate (Consepsis Scrub, Ultradent Products) in order to clean debris while disinfecting the area prior bonding. The veneers were bonded in pairs using the sequence of placement of \#8 and \#9, then \#7 and \#10, and finally \#6 and \#11. Ceramic restorations were treated with hydrofluoric acid surface (Porcelain Etch, Ultradent Products Inc.) for 20 seconds and then rinsed and dried copiously before being placed in an ultrasonic cleaner for 5 minutes for further cleaning. Silane (Monobond-S, Ivoclar Vivadent) was then applied for 60 seconds with rinsing and drying again. Cord \#00 was packed and the tooth substrate prepared for bonding with a traditional $32 \%$ phosphoric acid etching (Uni-Etch w/BAC, Bisco Dental; 30 seconds) and then rinsed and then fully dried (Fig. 4B). The primer was applied for 20 seconds (OptiBond FL, Kerr Dental), with excess removed by air, and then cured. The curing luting composite cement (Variolink Veneer Neutral Shade, Ivoclar Vivadent) was applied to ceramics \#8 and \#9, placed, and excess removed with a micro-brush and floss before light curing for 80 seconds total; 20 seconds on the mesial, incisal, facial, and the distal surface each. This process was repeated for teeth and veneers on \#7,\#10, \#6, and 

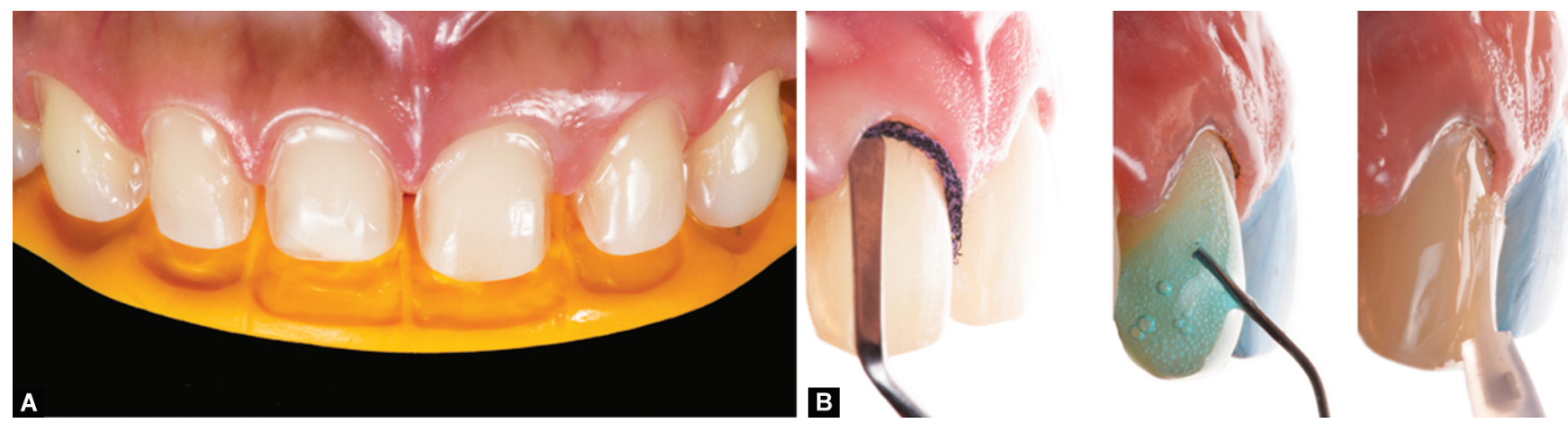

Figs 4A and B: (A) Final tooth preparations; (B) Bonding ceramic veneers
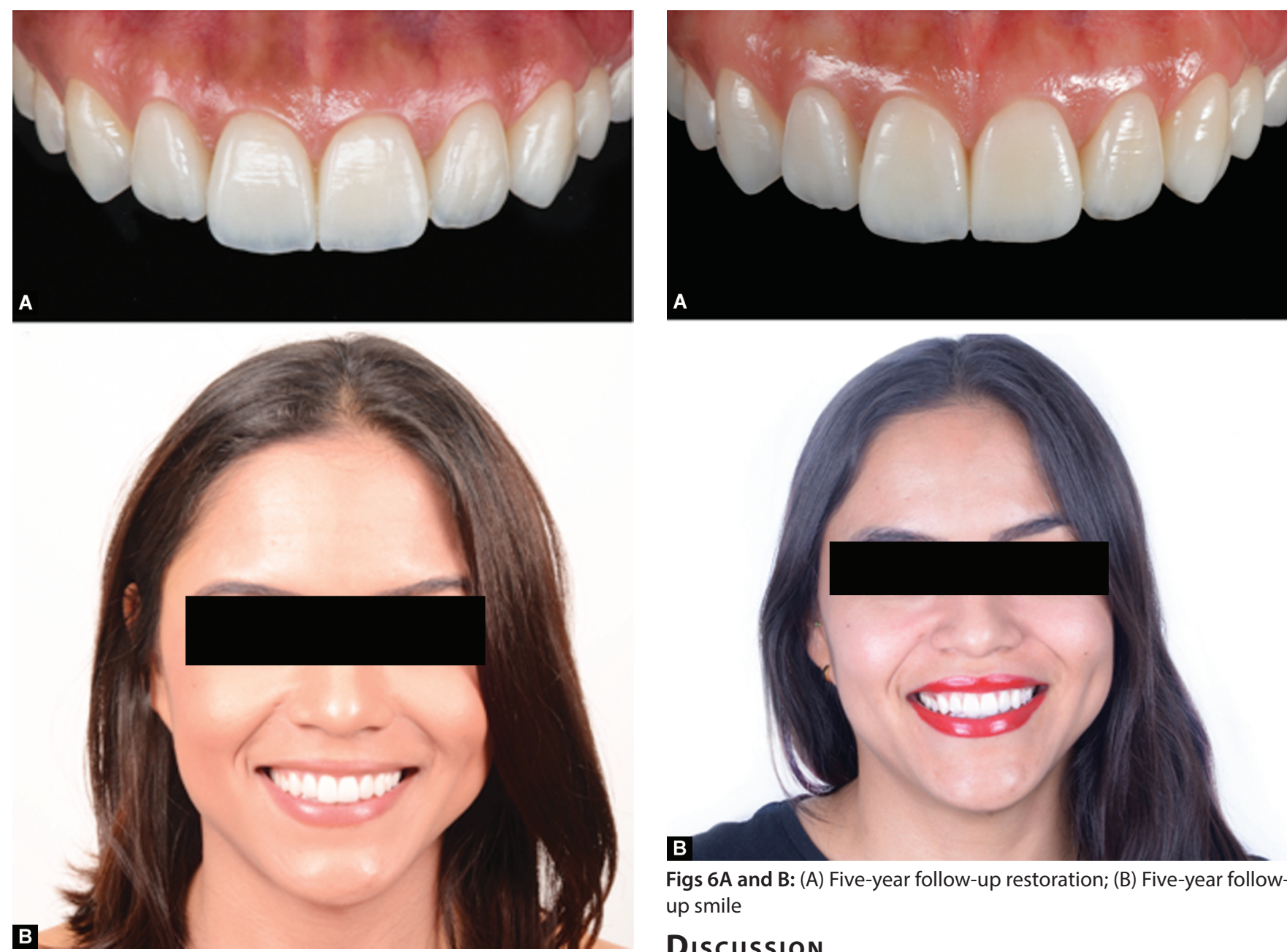

Figs 5A and B: (A) Final restorations; (B) Final smile

then \#11. Glycerin gel (Deox, Ultradent Products Inc.) was applied on the ceramic surface to prevent an oxygen inhibited layer and surfaces were then again light cured ( 20 seconds each). Excess resin cement was removed with a \#12 scalpel blade. Articulating paper (Articulating Paper Strips, Henry Schein) was used to check occlusion. The patient reported they were satisfied with the final esthetic results and reported no postoperative sensitivity (Fig. 5). The patient was still satisfied with the clinical results at the 5-year, periodic, follow-up (Fig. 6).

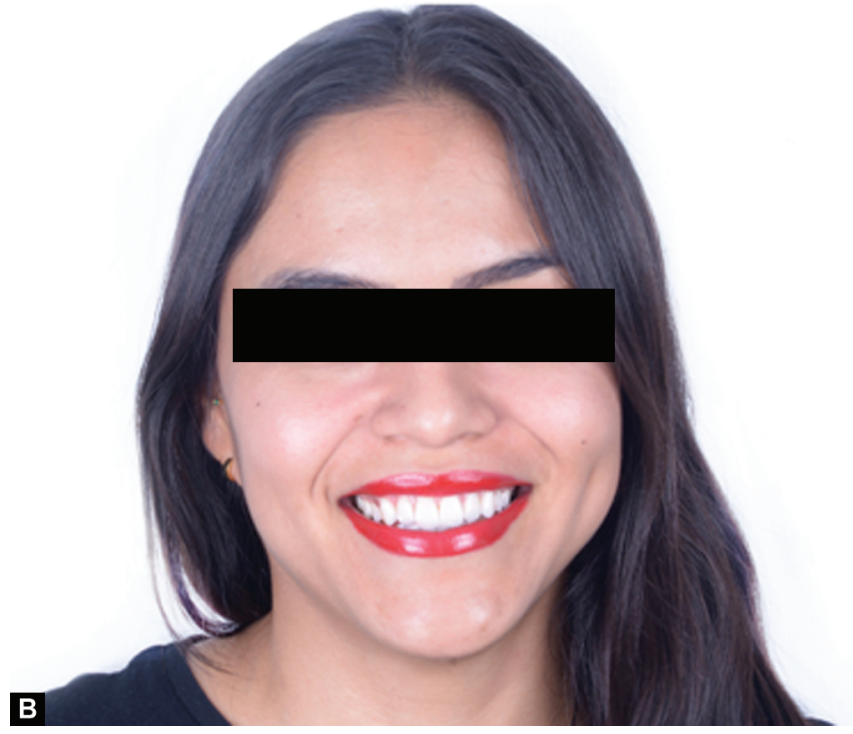

Figs $6 \mathrm{~A}$ and B: (A) Five-year follow-up restoration; (B) Five-year followup smile

\section{Discussion}

This clinical report of a 5-year follow-up of feldspathic ceramic veneers describes how diagnostic wax-ups, subsequent mock-up, and reduction guides can lead to good patient esthetics. A wax-up was first performed and then a mock-up was made. A putty matrix guide provided the patient with the ability to give feedbacks on the final restorations. Fabrication of reduction guides allow the clinician to measure individual preparation areas and control preparation so to be appropriate to the type of restoration desired and other clinical factors. ${ }^{28}$ Despite the fact that experienced clinicians may not view preparation guides as necessary, the authors recommend them. Excessive reduction of the tooth is a common mistake when 
guides are not used and may lead to dentin exposure and decreased bonding potential. Optimal tooth preparation can guarantee no disturbance of the periodontal health and structural integrity. ${ }^{30}$ Conversely, under reduction will cause the dental laboratory to fabricate over-contoured restorations.

Conservative preparations can provide sufficient remaining tooth structure to prepare for a full coverage crown in the future in case of veneer failure. Here, feldspathic porcelain (IPS e-max, Ivoclar Vivadent) was used to enable an ultrathin approach but the cementing strategy must also be robust to support such an approach. ${ }^{34}$ Since none of the current dental prosthesis can be guaranteed to last forever, it should be considered for both the clinician and the patient to have a very conservative and controlled tooth reduction. ${ }^{35}$ Similar to other case reports with excellent results, ${ }^{20}$ the cement strategy should also be considered for color stability and translucency. Digital restoration design, which has also shown predictable outcomes, ${ }^{36}$ may also assist throughout patient treatment. Long-term success of the restoration requires following well-defined protocols for restorative material selection, conservative tooth preparation, and bonding ceramic protocols.

\section{Conclusion}

Preparation guides allow optimal amounts of tooth reduction for veneer preparations. Diagnostic wax-ups and the subsequent mock-up allow the clinician to modify length and width of the teeth without any modification. Mock-ups then show patients a realistic representation of the future restoration. Reduction guides are recommended in order to provide adequate tooth reduction. When combined, the use of conservative tooth preparation and these techniques can improve the longevity of the restorations and demonstrate good esthetics at 5 years.

\section{Clinical Significance}

Conservative tooth preparation, reduction guides, and wax-ups may increase the lifespans of veneer restorations and demonstrate good esthetics at 5 years.

\section{References}

1. Van Meerbeek B, De Munck J, Yoshida Y, et al. Buonocore memorial lecture. Adhesion to enamel and dentin: current status and future challenges. Oper Dent 2003;28(3):215-235.

2. Aykor A, Ozel E. Five-year clinical evaluation of 300 teeth restored with porcelain laminate veneers using total-etch and a modified self-etch adhesive system. Oper Dent 2009;34(5):516-523. DOI: 10.2341/08038-C.

3. Nakabayashi N, Kojima K, Masuhara E. The promotion of adhesion by the infiltration of monomers into tooth substrates. J Biomed Mater Res 1982;16(3):265-273. DOI: 10.1002/jbm.820160307.

4. Dumfahrt $\mathrm{H}$, Schaffer $\mathrm{H}$. Porcelain laminate veneers. A retrospective evaluation after 1 to 10 years of service: part II clinical results. Int J Prosthodont 2000;13(1):9-18.

5. Peumans M, De Munck J, Fieuws S, et al. A prospective ten-year clinical trial of porcelain veneers. J Adhes Dent 2004;6(1):65-76.

6. Wakiaga J, Brunton P, Silikas N, et al. Direct versus indirect veneer restorations for intrinsic dental stains. Cochrane Database Syst Rev 2004(1):CD004347. DOI: 10.1002/14651858.CD004347.pub2.

7. Peumans $M$, Van Meerbeek B, Lambrechts P, et al. Porcelain veneers: a review of the literature. J Prosthet Dent 2000;28(3):163-177. DOI: 10.1016/s0300-5712(99)00066-4.

8. Magne $P$, Versluis A, Douglas WH. Effect of luting composite shrinkage and thermal loads on the stress distribution in porcelain laminate veneers. J Prosthet Dent 1999;81(3):335-344. DOI: 10.1016/s00223913(99)70278-7.

9. Magne P, Kwon KR, Belser UC, et al. Crack propensity of porcelain laminate veneers: a simulated operatory evaluation. J Prosthet Dent 1999;81(3):327-334. DOI: 10.1016/s0022-3913(99)70277-5.

10. Magne $P$, Douglas $W H$. Optimization of resilience and stress distribution in porcelain veneers for the treatment of crown-fractured incisors. Int J Periodontics Restorative Dent 1999;19(6):543-553.

11. Petridis $H$, Zekeridou $A$, Malliari $M$, et al. Survival of ceramic veneers made of different materials after a minimum follow-up period of five years: A systematic review and meta-analysis. Eur J Esth Dent 2012;7(2):138-152.

12. Lin T, Liu P, Ramp L, et al. Fracture resistance and marginal discrepancy of porcelain laminate veneers influenced by preparation design and restorative material in vitro. J Dent 2012;40(3):202-209. DOI: 10.1016/j. jdent.2011.12.008.

13. Lacy A, Wada C, Du W, et al. In vitro microleakage at the gingival margin of porcelain and resin veneers. J Prosthet Dent 1992;67(1):7-10. DOI: 10.1016/0022-3913(92)90038-c.

14. Della Bona A, Kelly J. A variety of patient factors may influence porcelain veneer survival over a 10-year period. J Evid-Based Dent $\operatorname{Pr}$ 2010;10(1):35-36. DOI: 10.1016/j.jebdp.2009.11.014.

15. Ozturk E, Bolay S, Hickel R, et al. Shear bond strength of porcelain laminate veneers to enamel, dentin and enamel-dentin complex bonded with different adhesive luting systems. J Dent 2013;41(2):97105. DOI: 10.1016/j.jdent.2012.04.005.

16. Strassler H. Minimally invasive porcelain veneers: indications for a conservative esthetic dentistry treatment modality. Gen Dent 2007;55(7):819-826.

17. Carpena G, Ballarin A, Aguiar J. New ceramics approach for contact lens. Odovtos-Int J Dent Sc 2015;17(1):12-18. DOI: 10.15517/ijds. v0i0.22040.

18. Trinkner T, Roberts M. Fluorapatite-leucite glass ceramic veneers for aesthetic anterior restorations. Pract Proced Aesthet Dentist 2001;13(1):37-41. DOI: 11301529.

19. Maicone P, Lammeti R, Raffaelli L. An overview of zirconia ceramics: basic properties and clinical applications. J Dent 2007;35(11):819-826. DOI: 10.1016/j.jdent.2007.07.008.

20. Soares $P$, Spini $P$, Carvalho V, et al. Esthetic rehabilitation with laminated ceramic veneers reinforced by lithium disilicate. Quintessence Int 2014;45(2):129-133. DOI: 10.3290/j.qi.a31009.

21. Conrad H, Seong W, Pesun I. Current ceramic materials and systems with clinical recommendations: A systematic review. J Prosthet Dent 2007;98(5):389-404. DOI: 10.1016/S0022-3913(07)60124-3.

22. Spear F, Holloway J. Which all-ceramic system is optimal for anterior esthetics? JADA 2008;139:19s-24s. DOI: 10.14219/jada. archive.2008.0358.

23. Gurel G, Sesma N, Calamita M. Influence of enamel preservation on failure rates of porcelain laminate veneers. Int J Periodontics Restorative Dent 2013;33(1):31-39. DOI: 10.11607/prd.1488.

24. Edelhoff D, Sorense J. Tooth structure removal associated with various preparation designs for anterior teeth. J Prosthet Dent 2002;87(5):503-509. DOI: 10.1067/mpr.2002.124094.

25. Piemjai M, Arksomnukit M. Compressive fracture resistance of porcelain laminate bonded to enamel or dentin with four adhesive systems. J Prosthodont 2007;16(6):457-464. DOI: 10.1111/j.1532849X.2007.00227.x.

26. Cotert H, Dundar M, Ozturk B. The effect of various preparation designs of the survival or porcelain laminate veneers. J Adhes Dent 2009;11(5):405-4-11.

27. Sadowsky S. An overview of treatment considerations for esthetic restorations: a review of the literature. J Prosthet Dent 2006;96(6):433-442. DOI: 10.1016/j.prosdent.2006.09.018.

28. Carlyle L, Richardson J. The diagnostic wax-up: as aid in treatment planning. Tex Dent J 1985;102(2):10-12.

29. Preston J. A systematic approach to the control of esthetic form. J Prosthet Dent 1976;35(4):393-402. DOI: 10.1016/0022-3913(76) 90006-8. 
30. Yuodelis R, Faucher R. Provisional restorations: an integrated approach to periodontics and restorative Dentistry. Dent Clin North Am 1980;24(2):285-303.

31. Phillips K, Morgan R. The acrylic occlusal plane guide: a tool for esthetic occlusal reconstruction. Compend Contin Educ Dent 2001;22(4):302-304.

32. Simon $\mathrm{H}$, Magne P. Clinically based diagnostic wax-up for optimal esthetics: the diagnostic mock-up. CDA J 2008;36(5):355-362.

33. Coachman C, Gurel G, Calamita M. The influence of tooth color on preparation design for laminate veneers from a minimally invasive perspective: a case report. Int J Periodontics Restorative Dent 2014;34(4):453-459. DOI: 10.11607/prd.1900.

34. Jurado CA, Tsujimoto A, Guzman LG, et al. Implant therapy with ultratranslucent monolithic zirconia restorations in the esthetic zone: a case report. Gen Dent 2020;68(1):46-49.

35. Jurado CA, Tsujimoto A, Tanaka K, et al.3D printed coping for intraoral evaluation: a clinical report. Quintessence Int 2019;50(7):534-538. DOI: 10.3290/j.qi.a42655.

36. Pimentel W, Teixeira M, Costa P, et al. Predictable outcomes with porcelain laminate veneers: a clinical report. J Prosthodont 2016;25(4):335-340. DOI: 10.1111/jopr.12413. 